\title{
AMPK phosphorylation by Ssp1 is required for proper sexual differentiation in fission yeast
}

\author{
Noelia Valbuena and Sergio Moreno* \\ Instituto de Biología Molecular y Celular del Cáncer CSIC, Salamanca University, Campus Miguel de Unamuno, Salamanca, 37007, Spain \\ *Author for correspondence (smo@usal.es) \\ Accepted 30 November 2011 \\ Journal of Cell Science 125, 2655-2664 \\ (C) 2012. Published by The Company of Biologists Ltd \\ doi: $10.1242 / j c s .098533$
}

\section{Summary}

The AMP-activated protein kinase (AMPK) is a central regulator of cellular energy homeostasis, which, in response to a fall in intracellular ATP levels, activates energy-producing pathways and inhibits energy-consuming processes. Here, we report that fission yeast cells lacking AMPK activity are unable to advance entry into mitosis in response to nitrogen starvation and cannot undergo proper G1 arrest and cell differentiation. We also show that AMPK is important in the promotion of the nuclear localization and accumulation of the Ste11 transcription factor. As in animal cells, the fission yeast CaMKK ortholog (Ssp1) phosphorylates and activates the catalytic subunit of AMPK (Ssp2) in its activation loop (Thr189) when cells are starved for nitrogen or glucose. Interestingly, we found that the phosphorylation of Ssp2 on Thr189 is required for nuclear accumulation of AMPK. Our data demonstrate the existence of a signal transduction pathway activated by nutrient starvation that triggers Ssp2 phosphorylation and AMPK redistribution from the cytoplasm to the nucleus. This pathway is important to advance fission cells into mitosis and to establish a timely pre-Start G1 cell cycle arrest for mating.

Key words: S. pombe, Cell cycle, Differentiation, AMPK, Ssp2, Ssp1

\section{Introduction}

The AMP-activated protein kinase (AMPK) is a conserved multisubstrate kinase that is activated in response to a variety of metabolic stresses to regulate cell energy homeostasis. AMPK activity helps to adapt the cell metabolism to match available energy resources (Hardie, 2008). Its activation depends both on its phosphorylation on Thr172 by upstream kinases (Hawley et al., 2003) and on the cellular ATP and AMP concentration (Hardie, 2003). AMP is an allosteric activator of AMPK and makes AMPK a better substrate for the upstream kinase. The tumour suppressor LKB1 and CaMKK (calcium/calmodulin-dependent protein kinase kinase) activate mammalian AMPK by phosphorylation of the $\alpha$-subunit at a specific threonine residue (Thr172) (Hawley et al., 2005; Hurley et al., 2005; Woods et al., 2005). AMPK phosphorylation causes at least a 100-fold activation, whereas allosteric activation by AMP is about 10fold. AMPK is stimulated by AMP and ADP and is inhibited by high concentrations of ATP (Hardie, 2011; Hawley et al., 1996; Xiao et al., 2011). When intracellular ATP levels drop, active AMPK inhibits ATP-consuming processes. When ATP levels increase, inactive AMPK allows energy usage for biosynthetic pathways and processes that consume ATP. This is how eukaryotic cells adapt their metabolism to the intracellular energy status (Hardie et al., 2006).

Fission yeast AMPK forms a $\alpha \beta \gamma$ heterotrimer between the catalytic subunit Ssp2 and the $\beta \gamma$ regulatory subunits Amk 2 and Cbs2 (Townley and Shapiro, 2007). In general, the $\alpha$-subunits include a typical serine-threonine kinase domain characteristic of AMPKs. The $\beta$-subunits are required for trimer assembly and the subcellular localization of the AMPK complex (Vincent et al., 2001; Warden et al., 2001). The heart of AMPK regulation by adenylate binding is thought to reside in the $\gamma$-subunit (Adams et al., 2004), where ATP or AMP binds. Another catalytic subunit, Ppk9, has been described in Schizosaccharomyces pombe. Ppk9 also interacts with Amk2 and Cbs2 (Hanyu et al., 2009). In $S$. pombe, ADP is also able to bind to the $\beta$-subunit, suggesting a possible role for ADP in regulating AMPK (Jin et al., 2007). Ssp2 (the $\alpha$-subunit) contains the ubiquitin-associating UBA domain immediately adjacent to the kinase domain (Hanyu et al., 2009). This feature resembles the mammalian AMPKrelated kinases MARK or BRSK, which are the substrates of LKB1 (Jaleel et al., 2006). Recently, Ssp1 has been described as the orthologue of CaMKK protein in $S$. pombe that contains the kinase domain and a putative CBD region (calmodulin-binding domain) (Hanyu et al., 2009). Interestingly, Ssp1 and Ssp2 were described originally to be required for growth polarity and actin localization in fission yeast (Matsusaka et al., 1995).

In spite of the importance of AMPK in signaling the cellular energy status, and despite the simplicity of $S$. pombe as a eukaryotic model organism, very little is known about AMPK regulation in fission yeast. Here we show that the AMPK complex (Ssp2-Amk2-Cbs2) is needed for the correct transition from cell proliferation to cell differentiation under low-energy conditions and that it is regulated by two different mechanisms: phosphorylation of the catalytic subunit and subcellular localization of the AMPK complex.

\section{Results}

Fission yeast AMPK is required for proper cell cycle exit and sexual differentiation

Crystal structure analysis revealed that fission yeast AMPK is comprised of $\alpha-\beta$ - and $\gamma$-subunits corresponding to Ssp2 
( $\alpha$-subunit), Amk2 ( $\beta$-subunit) and Cbs2 ( $\gamma$-subunit) (Townley and Shapiro, 2007). To check whether the three AMPK subunits indeed interact in vivo, cells co-expressing epitope-tagged versions of Ssp2 (Ssp2-HA) and Amk2 (Amk2-TAP) or Cbs2 (Cbs2-TAP) were generated. TAP-epitope-tagged proteins were pulled down from extracts obtained from exponentially growing yeast cells using IgG-Sepharose beads and the presence of Ssp2HA in the pellets was examined by western blot. Ssp2 was pulled down with Amk2 or with Cbs2 (Fig. 1A). We also were able to detect Amk2 and Cbs2 in pull downs of Ssp2. These results indicated that Ssp2 interacts physically with Amk2 and Cbs2 in vivo, showing that AMPK is indeed a $\alpha \beta \gamma$ heterotrimer and that these three subunits interact even under optimal growth conditions. This result is in agreement with previous mass spectrometry analyses reported by (Hanyu et al., 2009).

Ssp2 (the catalytic $\alpha$-subunit of AMPK) was identified in a systematic deletion analysis of fission yeast protein kinases. Loss of the $\operatorname{ssp} 2$ gene in a homothallic $h^{90}$ strain showed partial iodine staining in the sporulation medium, indicating a defect in cell cycle exit and/or sexual differentiation (Bimbó et al., 2005). Therefore, we analyzed the role of the three subunits of AMPK in mating and sexual differentiation. In animal cells, AMPK is activated by phosphorylation by two upstream kinases: LKB1 and CaMKK. The CaMKK orthologue in $S$. pombe is Ssp1 and it can be considered functionally similar to mammalian LKB1 (Hanyu et al., 2009). Accordingly, we also analyzed the mating efficiency of the ssp1 1 mutant. As shown in Fig. 1B,C, deletion of the genes encoding any of the three subunits of AMPK or of Ssp1 showed a similar phenotype, a decrease in the percentage of zygotes (Fig. 1B), and a reduction in staining with iodine vapour (Fig. 1C), indicating that mating efficiency is diminished in all of them. We therefore conclude that each of the three subunits of AMPK and Ssp1 are required for proper sexual differentiation in $S$. pombe.

When fission yeast cells are starved of nitrogen, G2 cells are advanced into mitosis and eventually arrest in the G1 phase of the cell cycle after two rounds of division with a small cell size (Fantes and Nurse, 1977). This G1 arrest is a prerequisite for mating and meiosis (Egel and Egel-Mitani, 1974; Nurse and Bissett, 1981). To study whether AMPK plays a role in cell cycle exit and G1 arrest, we compared the FACS profile and the cell size of the wild-type and the ssp2 $\Delta$ mutant cells after nitrogen starvation. Flow cytometry analysis revealed that ssp 2 cells arrested in G1 more slowly and showed a G2 population with a larger size than the wild-type cells (see Fig. 1D, 2-6 hours). However, cell cycle arrest before $\mathrm{S}$ phase was not completely abolished and the cells eventually arrested with a 1C DNA content (Fig. 1D, 1014 hours). After nitrogen withdrawal, cells arrested in G1 were small. To test whether there were differences in the decrease in cell

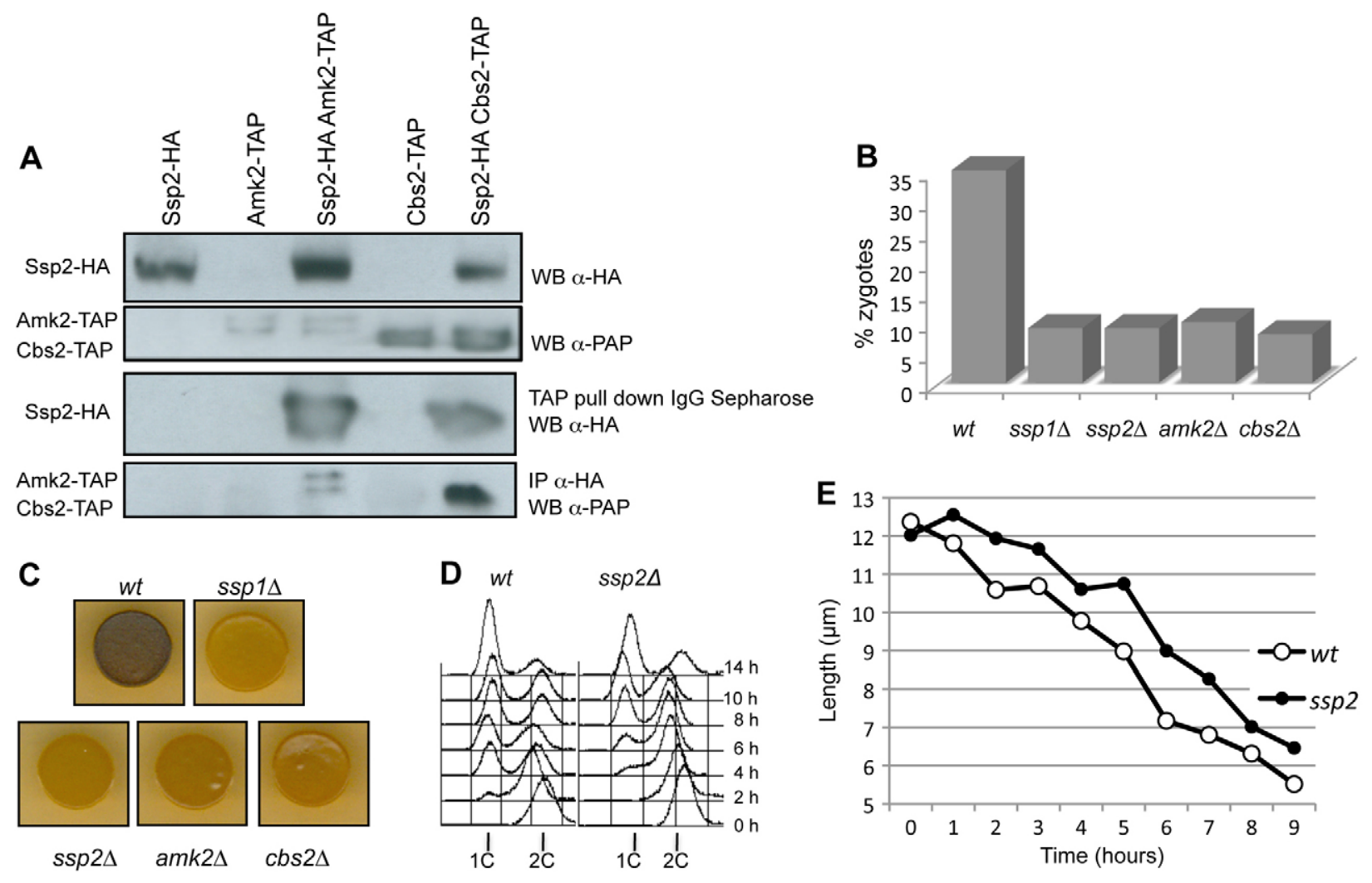

Fig. 1. Effects of the AMPK complex on G1 cell cycle arrest and sexual differentiation. (A) The catalytic subunit of AMPK (Ssp2) interacts in vivo with the $\beta$ - (Amk2)- and the $\gamma$ - (Cbs2) subunits. Amk2-TAP and Cbs2-TAP were pulled down using IgG-Sepharose and Ssp2-HA was detected by blotting. Ssp2-HA was immunoprecipitated with anti-HA antibodies and Amk2-TAP and Cbs2-TAP were detected by western blot. (B,C) Wild-type, ssp1, ssp2, amk2 and cbs2 mutant cells of the opposite mating were grown to mid-exponential phase, spotted onto malt extract plates and incubated for 24 hours at $25^{\circ} \mathrm{C}$. The percentage of zygotes was determined (B) and the spores were stained with iodine vapour (C). (D,E) Wild-type and $\operatorname{ssp} 2$ cells were grown to mid-exponential phase in minimal medium at $25^{\circ} \mathrm{C}$, washed several times, and incubated in minimal medium lacking nitrogen. Wild-type and $\operatorname{ssp} 2$ mutant cells were harvested at the indicated times and the DNA content and cell size were determined by flow cytometry (D) and microscopy (E). To measure the size of dividing cells, we stained the cell wall with calcofluor to identify dividing cells containing a septum. 
length, we measured wild-type and ssp $2 \Delta$ dividing cells every hour after nitrogen starvation. ssp $2 \Delta$ cells showed a delay in the reduction in cell size compared with wild-type cells (Fig. 1E; supplementary material Fig. S1 for additional images taken every hour during the nitrogen starvation experiment), suggesting that AMPK is required for proper advance entry into mitosis in nitrogen-starved cells and arrest in G1 before Start.

\section{Fission yeast AMPK regulates Ste11 levels under nitrogen deprivation}

The Ste11 transcription factor is a key regulator of the switch from mitotic cell division to sexual differentiation (Sugimoto et al., 1991). During mitotic growth, Ste 11 is inhibited by Cdk1 phosphorylation (Kjaerulff et al., 2007). Nitrogen limitation inactivates $\mathrm{Cdk} 1$ and induces the expression of ste $11^{+}$mRNA and that of many other genes required for conjugation and sporulation in fission yeast (Sugimoto et al., 1991).

Because AMPK is required for proper sexual differentiation, we compared the levels of ste $11^{+}$mRNA and protein in wild-type and ssp $2 \Delta$ mutant cells. As shown in Fig. $2 \mathrm{~A}$, ste $11^{+}$mRNA induction was reduced in ssp $2 \Delta$ cells, suggesting that AMPK activity was required for full induction of ste $^{+}$mRNA transcription. We also compared Ste11 protein levels after nitrogen starvation in wild-type and ssp $2 \Delta$ cells by western blot. As shown in Fig. 2B, the levels of Ste11 protein were clearly reduced in $\operatorname{ssp} 2 \Delta$ cells. These observations indicated that AMPK is required for the proper induction and accumulation of Ste11 transcription factor under nitrogen deprivation, which is probably the reason why $\operatorname{ssp} 2$ cells do not mate correctly. To corroborate this, we overexpressed ste $11^{+} \mathrm{mRNA}$ in $\operatorname{ssp} 2 \Delta$ cells and found that $s t e 11^{+}$overexpression rescued the sterility phenotype of the ssp2A mutant (Fig. 2C), confirming that AMPK is acting upstream of Ste11.

\section{How can AMPK regulate Ste11 levels after nitrogen starvation?}

The expression of ste $^{+} 1^{+}$is under the regulation of the cAMPdependent protein kinase (PKA). PKA plays a key role in sensing the nutritional environment, promoting cell growth and repressing sexual differentiation by inhibiting and preventing the nuclear accumulation of the Rst 2 transcription factor that is

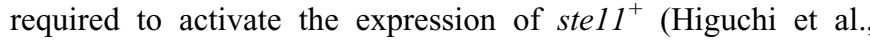
2002). To test whether the lack of AMPK activity was able to delocalize Rst2 to the cytoplasm to prevent $s t e 11^{+}$transcription, we looked at Rst2-GFP localization by fluorescent microscopy in wild-type and ssp $2 \Delta$ mutant cells. As shown in supplementary material Fig. S2, Rst2-GFP localized to the nucleus, both in the presence and in the absence of nitrogen, in wild-type and in ssp2 4 mutant cells, indicating that Ssp2 does not regulate Rst2 subcellular localization. This result might explain why ste11 mRNA and protein levels were not completely abolished after nitrogen starvation (Fig. 2A,B).

We next wondered whether Ste11 was active in ssp2 4 mutant cells. In wild-type exponentially growing cells, Ste11 was present at low levels and was pancellular (Fig. 3A, time 0 hours). Ste11 stimulates its own transcription (Kunitomo et al., 2000) when it accumulates in the nucleus after nitrogen starvation, generating a positive-feedback loop (Qin et al., 2003). We analyzed Ste11-GFP subcellular localization in wild-type and ssp2 $\Delta$ mutants after nitrogen starvation and determined the percentage of cells with Ste11 nuclear staining. As shown in Fig. 3A,B, the percentage of cells with Ste11 protein present in the nucleus was higher in the wild type than in the $\operatorname{ssp} 2 \Delta$ mutant. Moreover, the intensity of the Ste11-GFP nuclear signal was higher in the wild type than in the ssp2 $\triangle$ mutant, suggesting that AMPK activity is required either to increase Ste11 protein levels (Fig. 2) or, alternatively, that AMPK promotes nuclear accumulation of Ste11 in nitrogen-starved cells.

\section{AMPK is essential in the regulation of the response to nitrogen starvation}

AMPK is activated when the ATP:ADP ratio falls below a certain threshold level in the cell, switching on ATP-generating catabolic pathways and switching off ATP-consuming anabolic processes, to adapt to the nutritional stress. Because Ssp2 seems to be required for the nuclear accumulation of the Ste11 transcription factor, we performed microarray analysis in wild-type and ssp $2 \Delta$ cells after nitrogen starvation. Supplementary material Tables S1 and S2 list the genes that were not properly induced and

A

$w t$

$\operatorname{ssp} 2 \Delta$

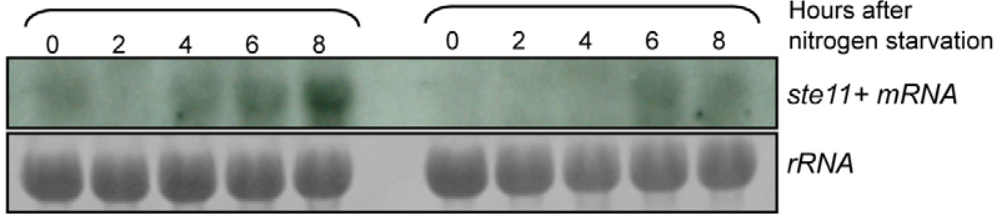

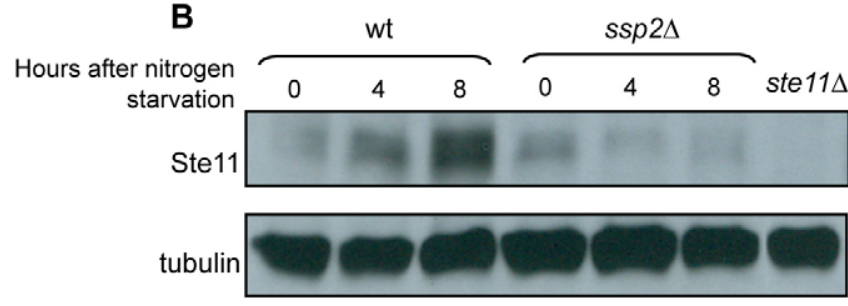

C

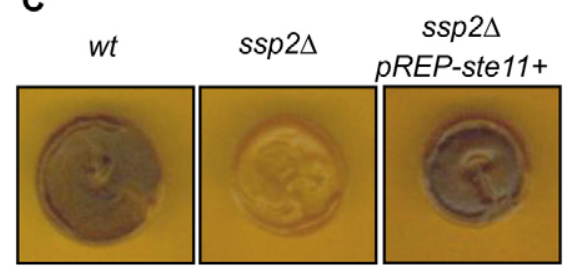

Fig. 2. Ste11 expression is impaired in the ssp2 mutant. (A) Northern blot showing levels of ste $11^{+}$mRNA after nitrogen starvation (hours) in wild-type and ssp 24 cells. rRNA was used as loading control. (B) Western blot analysis showing Ste11 protein levels in wild-type and ssp2 cells after nitrogen starvation (in hours). Tubulin was used as a loading control. (C) Mating efficiency of wild-type, ssp2 $\Delta$ and $s s p 2 \Delta$ pREP-stel $1^{+}$cells. Cells were grown to mid-exponential phase, spotted onto malt extract plates, and stained with iodine vapour. 
A

Oh

GFP

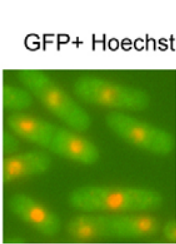

$2 \mathrm{~h}$
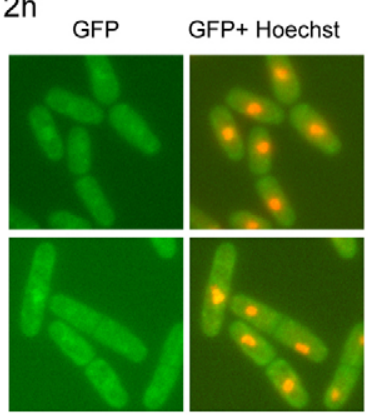

6h GFP

GFP+ Hoechst
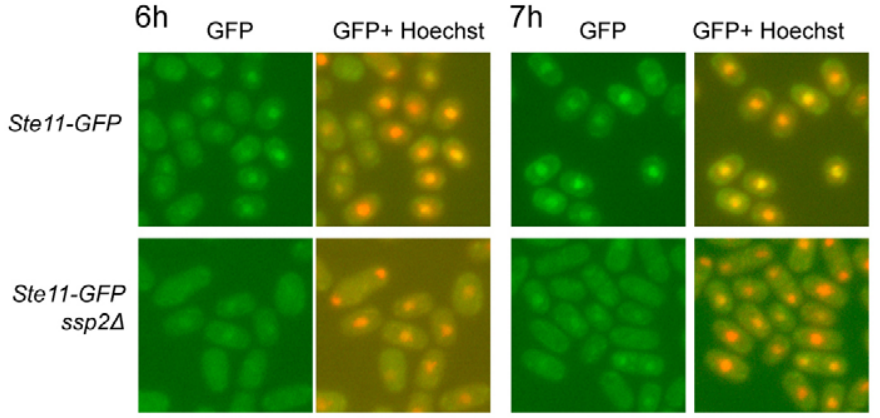

$4 h$
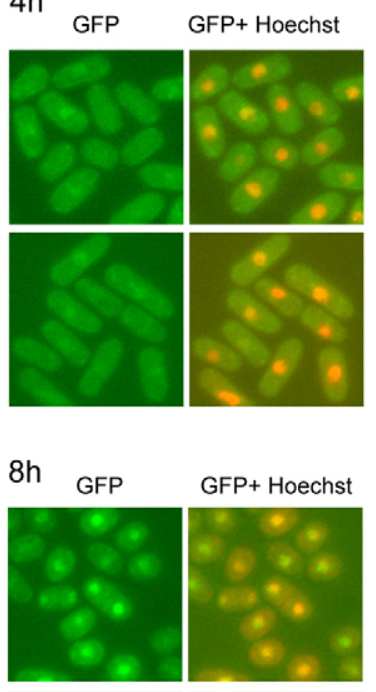

$5 \mathrm{~h}$
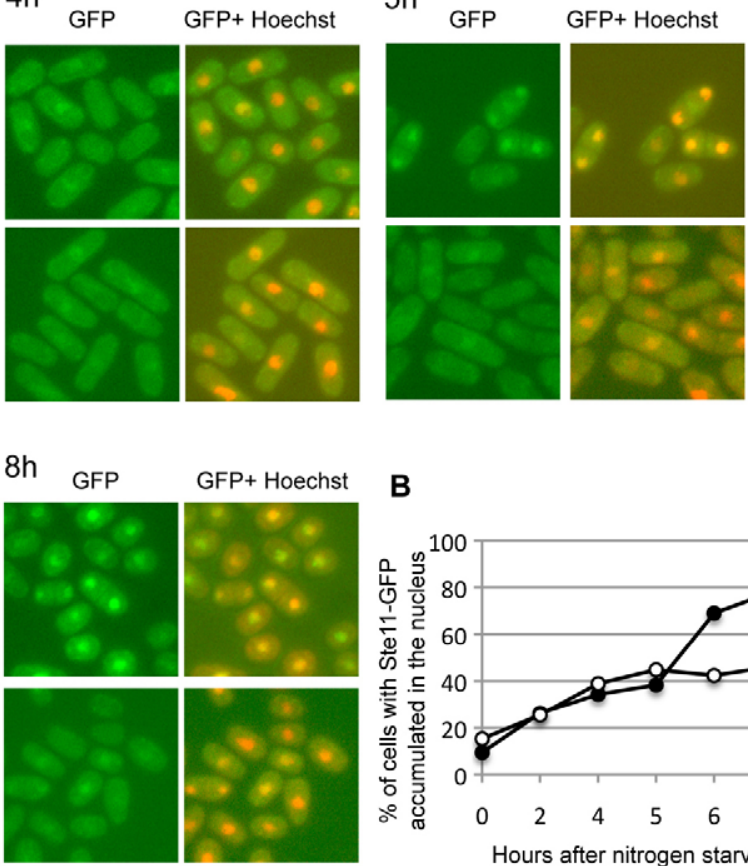

B

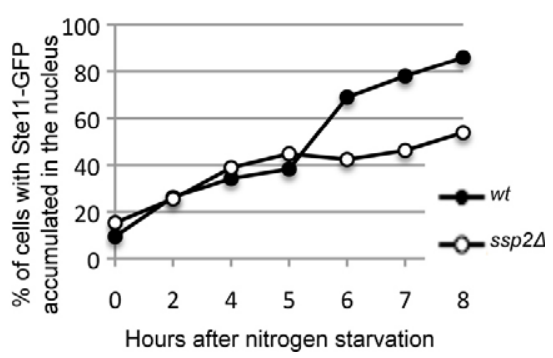

Fig. 3. Nuclear localization of Ste11. Ste11-GFP was observed by fluorescence microscopy in wild-type and ssp2 $\Delta$ cells during exponential growth and at different times (in hours) after nitrogen starvation. (A) Ste11-GFP is pancellular during exponential growth and accumulates in the nucleus after nitrogen starvation in wild-type cells. In ssp $2 \Delta$ cells, the amount of Ste11-GFP localized in the nucleus is reduced, especially after 5 hours. Nuclei are stained with Hoechst 33342. (B) Percentage of cells with nuclear staining of Ste11-GFP at different times after nitrogen starvation. After 5 hours, the percentage of ssp2 $\Delta$ cells accumulating Ste11-GFP in the nucleus did not increase to the same intensity as in wild-type cells. Scale bar: $10 \mu \mathrm{m}$.

repressed in ssp2 4 cells under nitrogen starvation. Ssp2 was required to repress the transcription of genes involved in metabolic processes that require ATP, such as DNA replication and translation (including histones and translation factors) and to activate the genes required to complete the current cell cycle, such as regulators of mitosis and cytokinesis and the genes required for sexual differentiation (including stell and its targets). In sum, AMPK drives the adaptation to nitrogen starvation by means of activating completion of the current cell cycle (entry into mitosis and cytokinesis) and sexual differentiation (G1 arrest, mating and spore formation). Importantly, the fact that Ste11 and its targets were not properly induced in ssp $2 \Delta$ cells confirms the notion that Ssp2 would be essential for Ste11 activity, as mentioned above. Thus, after nitrogen starvation AMPK becomes active and drives the cell through mitosis and cell division to promote sexual differentiation.

\section{AMPK moves into the nucleus upon nitrogen starvation}

In eukaryotic cells, AMPK is present both in the nucleus and in the cytoplasm. Environmental stress regulates the intracellular localization of AMPK; for example, in mammalian cells AMPK accumulates in the nucleus upon recovery from heat shock or oxidative stress (Kodiha et al., 2007). However, the mechanism that regulates the intracellular localization of AMPK is poorly understood.

To examine AMPK subcellular localization, we tagged each subunit of AMPK with green fluorescent protein (GFP) in the chromosome. We determined the subcellular localization of Ssp2-GFP, Amk2-GFP and Cbs2-GFP in complete minimal medium and after glucose or nitrogen starvation (Fig. 4). All three subunits showed the same localization pattern. In complete minimal medium, they were excluded from the nucleus and were only present in the cytoplasm. However, upon glucose or nitrogen starvation AMPK was evenly distributed in the nucleus and the cytoplasm. This finding suggested that inactive AMPK is excluded from the nucleus and, upon activation by glucose or nitrogen starvation, part of the AMPK moves into the nucleus.

\section{Ssp2 is phosphorylated in vivo by Ssp1}

Activation of human AMPK depends on a marked decrease in intracellular ATP levels and phosphorylation by the upstream kinase LKB1 at Thr172 (Thr189 in S. pombe), located in the activation loop of the catalytic $\alpha$-subunit. This phosphorylation is quantitatively more important (100-fold) than the allosteric activation by AMP (10-fold) (Hawley et al., 1996). To analyze the phosphorylation status of each AMPK subunit in fission yeast, protein extracts were prepared after nitrogen starvation, subjected to SDS-PAGE, and examined by western blot. No clear shifts in mobility were observed for Amk2 or Cbs2 (data not shown). However, Ssp2 showed three slow-migrating bands that disappeared after treatment with protein phosphatase (Fig. 5A), indicating that Ssp2 is hyperphosphorylated in vivo, as described previously (Hanyu et al., 2009). The upper band accumulated in both nitrogen- and in glucose-starved cells (Fig. 5A), suggesting that Ssp2 is hyperphosphorylated under nutritional stress.

To check whether the CaMKK protein kinase orthologue (Ssp1) was responsible for this phosphorylation, we tagged wildtype Ssp2 and a mutant in which Thr189 had been replaced by alanine [Ssp2(T189A)] with HA, both in the wild-type and in the 


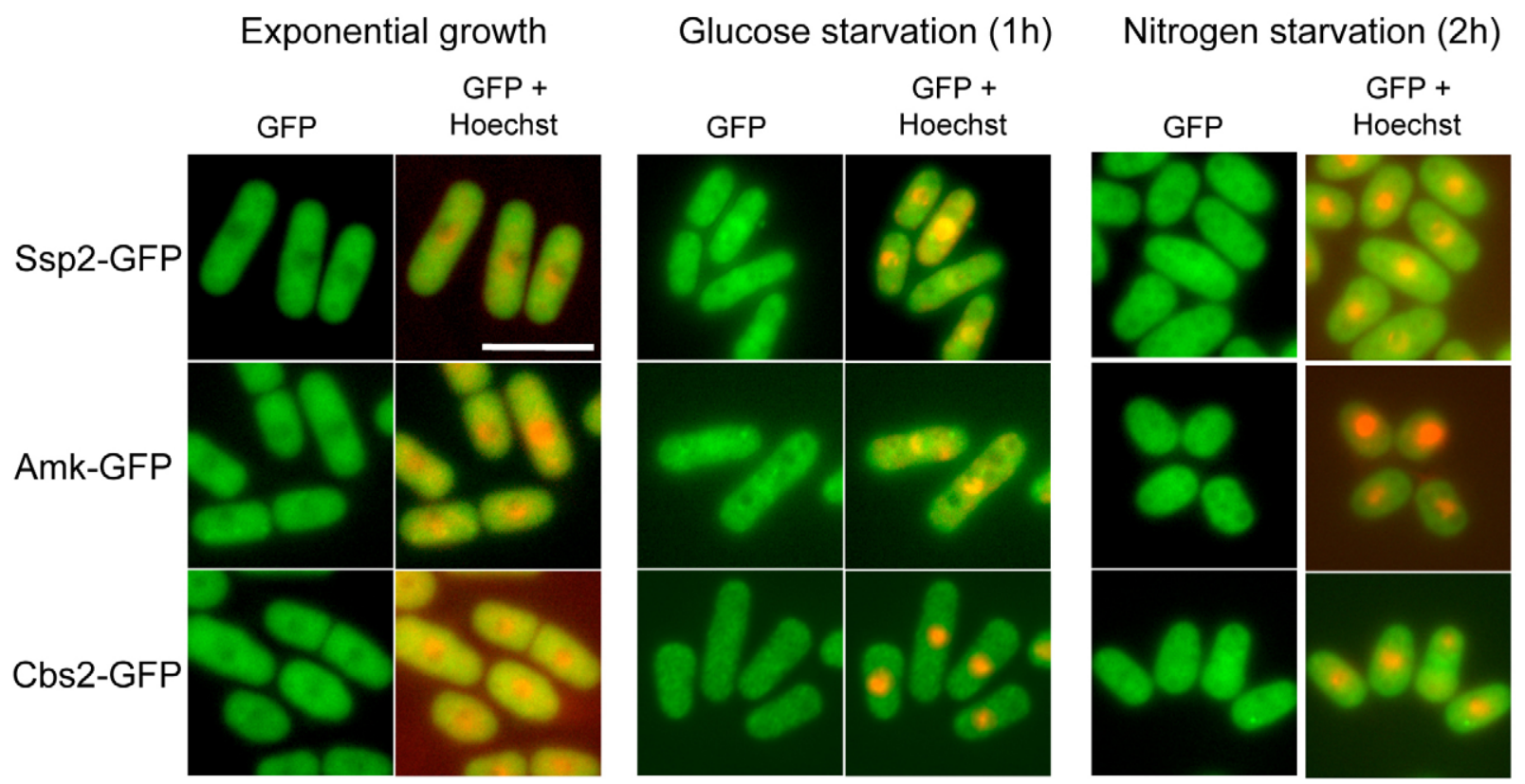

Fig. 4. AMPK subcellular localization. Ssp2-GFP, Amk2-GFP and Cbs2-GFP were observed by fluorescence microscopy in exponentially growing wild-type cells and after glucose $(1 \mathrm{~h})$ and nitrogen starvation $(2 \mathrm{~h})$. All the subunits of the AMPK complex show a cytoplasmic localization in exponentially growing cells, but under nutritional stress they also localize to the nucleus. Nuclei are stained with Hoechst 33342. Scale bar: $10 \mu \mathrm{m}$.

ssp14 mutant. The Ssp2 band with the slowest migration disappeared both in cells lacking Ssp1 and in cells expressing Ssp2(T189A) (Fig. 5B), suggesting that Ssp2 is phosphorylated in vivo on Thr189 and that Ssp1 could be the kinase responsible for this phosphorylation.

\section{Ssp2 phosphorylation is required for its nuclear entry in nutritionally stressed cells}

The results described above suggested that in cells stressed for nutrients Ssp2 is phosphorylated on Thr189 by Ssp1 and moves from the cytoplasm to the nucleus (Fig. 4, Fig. 5A,B). Next, we tested whether phosphorylation of Ssp2 is the signal for its nuclear localization after nutritional stress or whether AMPK is able to localize to the nucleus independently of its phosphorylation status. We analyzed Ssp2-GFP localization after nitrogen starvation (Fig. 6A) in wild-type and ssp1 $\Delta$ mutant cells. After 1 hour of nitrogen starvation, we were able to observe Ssp2-GFP inside the nucleus in wild-type cells, but not in the $\operatorname{ssp} 1 \triangle$ mutant, indicating that Ssp2 phosphorylation by $\mathrm{Ssp} 1$ is required for the nuclear localization of Ssp2 after nitrogen deprivation.

We also examined Ssp2-GFP localization after glucose starvation. In this case, the nuclear translocation of Ssp2 occurred even faster than under nitrogen starvation; by 30 minutes we were able to observe Ssp2-GFP in the nucleus (Fig. 6B). Therefore, Ssp1 is essential for the nuclear localization of Ssp2-GFP after glucose starvation. This result shows that Ssp2 phosphorylation by $\mathrm{Ssp} 1$ is required to trigger the nuclear
A

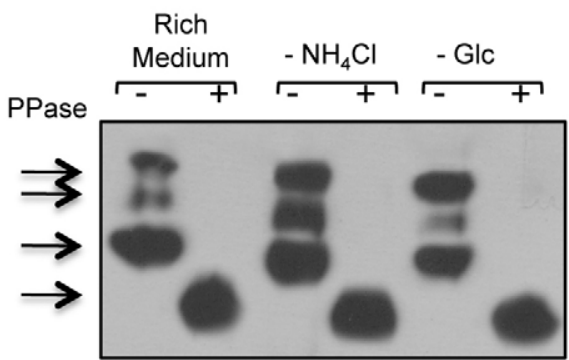

B

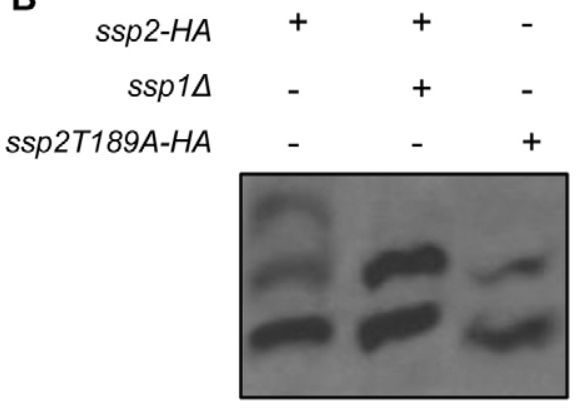

Fig. 5. Ssp2 phosphorylation. (A) Cells expressing Ssp2-HA were grown to mid-exponential phase in minimal medium ( $2 \%$ glucose) and then shifted to medium with low glucose $(0.2 \%$ glucose $)$ and medium lacking nitrogen. Cells were collected under these three different nutritional conditions. Protein extracts were immunoprecipitated with HA antibody and resolved by SDS-PAGE in the presence of a phos tag. Each sample was preincubated with alkaline phosphatase (PPase, + ) or only in buffer (-). (B) Wild-type cells, ssp14 cells expressing Ssp2-HA and wild-type cells expressing Ssp2(T189A)-HA were collected and protein extracts were resolved by SDS-PAGE in the presence of a phos tag. The most hyperphosphorylated band of Ssp2-HA is not observed in ssp1 $\Delta$ or in the inactive version of Ssp2. 


\section{A Nitrogen starvation}

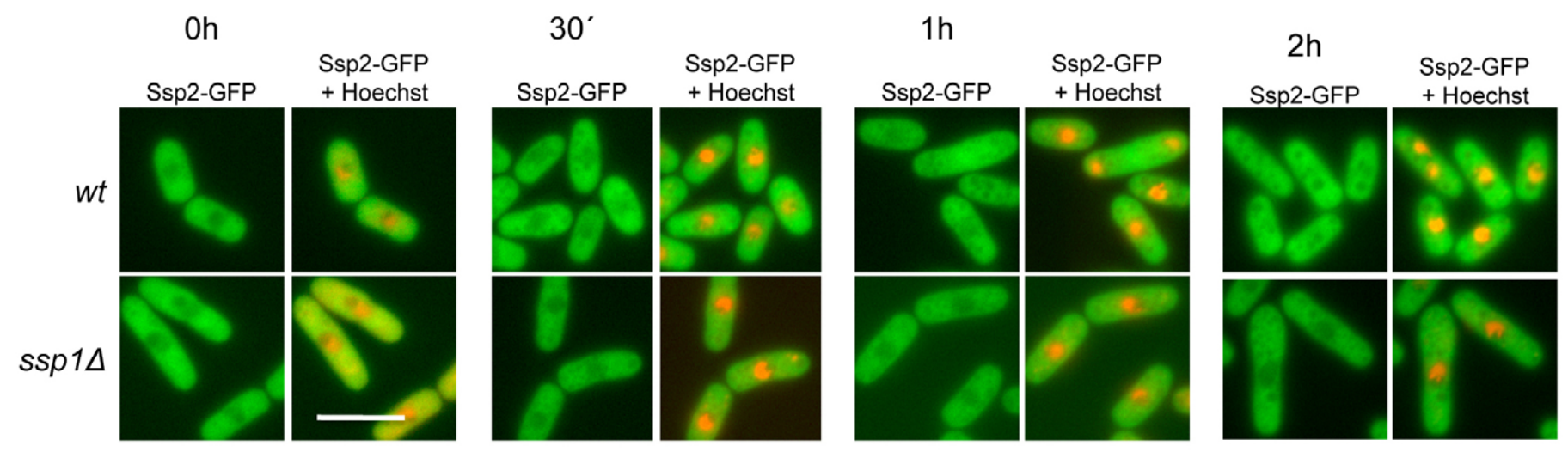

B Glucose starvation

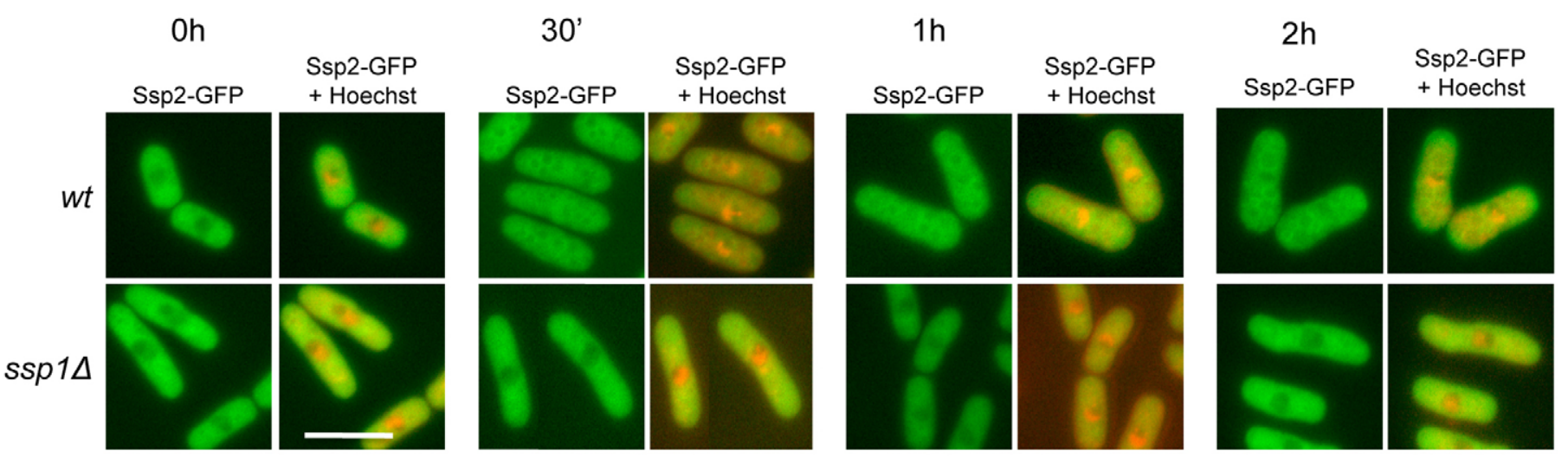

Fig. 6. Subcellular localization of Ssp2. Ssp2-GFP was observed by fluorescence microscopy in wild-type and $s s p 1 \Delta$ cells during exponential growth

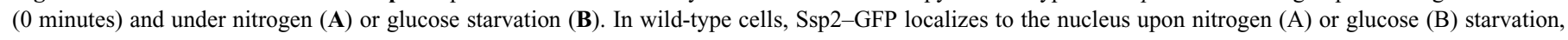

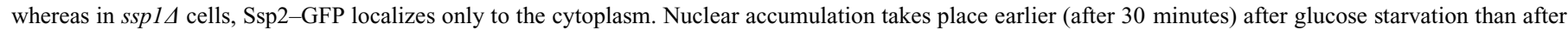
nitrogen starvation (1 hour). Scale bars: $10 \mu \mathrm{m}$.

accumulation of the former upon nitrogen or glucose starvation, and that if Ssp2 is not phosphorylated it remains in a cytoplasmic localization, regardless of the nutritional conditions of the cell.

\section{Is Thr189 phosphorylation essential for AMPK activity?}

Finally, we tested whether phosphorylation of Ssp2 by Ssp1 in $S$. pombe is required for AMPK activity in vivo. We integrated inactive (ssp2-T189A) and phosphomimetic (ssp2-T189E) ssp2 alleles expressed under their own promoters in the ssp 24 background. As a control, we used the $s s p 2^{+}$gene. Wild-type cells responded to nitrogen starvation by advancing entry into mitosis and cell division, generating small G1 cells (Egel and Egel-Mitani, 1974; Nurse and Bissett, 1981). ssp2 $\triangle$ cells arrested in G1 with a slow kinetic and with a larger cell size than wildtype cells (see also Fig. 1D,E; supplementary material Fig. S1). We tested the cell size of the active and the inactive forms of ssp2 after nitrogen starvation (Fig. 7A). $s s p 2^{+}$cells or cells expressing the phosphomimetic version of Ssp2 [ssp2(T189E)] arrested in G1 with a cell size similar to that of wild-type cells. However, cells expressing the inactive form of Ssp2 [ssp2(T189A)] showed a larger cell size, similar to that of $\operatorname{ssp} 2 \Delta$ cells. Moreover, Ssp2(T189A)-GFP was localized in the cytoplasm and no nuclear localization was observed (Fig. 8), indicating that Thr189 phosphorylation is essential for nuclear accumulation of Ssp2 under glucose and nitrogen starvation. We also determined the mating efficiency, as an indicator of AMPK activity (Fig. 7B,C), and found that ssp2 4 cells or cells expressing the inactive form of Ssp2 [ssp2(T189A)] underwent a reduction in the mating rate compared with wild-type cells. However, $s s p 2^{+}$or cells expressing phosphomimetic Ssp2 [ssp2(T189E)] underwent sexual differentiation at a similar level to wild-type cells. We also tested whether cells expressing ssp2(T189E) were able to bypass the Ssp1 requirement to promote sexual differentiation. We observed that ssp2(T189E) ssp1 4 cells were able to rescue the sterility phenotype of ssplA cells (Fig. 7D,E). These results strongly suggest that the phosphorylation of Ssp2 on Thr189 by Ssp1 is essential for AMPK to carry out proper cell cycle exit and sexual differentiation in fission yeast.

\section{Discussion}

The control of cell growth and differentiation is a complex process governed by nutrients and growth factors that are sensed by specialized signal transduction pathways. The AMP-activated protein kinase functions as a canonical suppressor of cell 
A
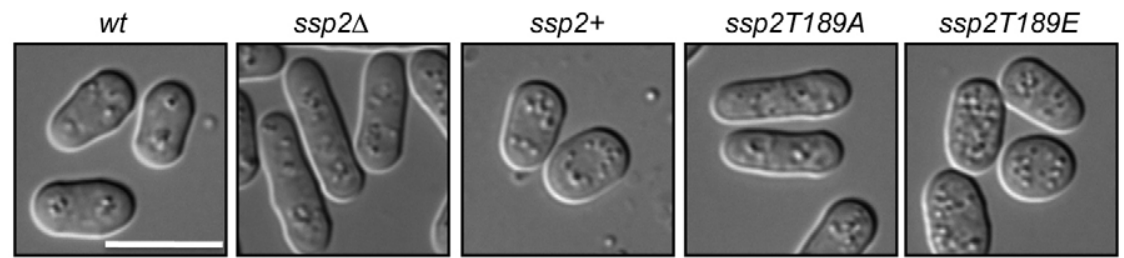

B

\section{D}

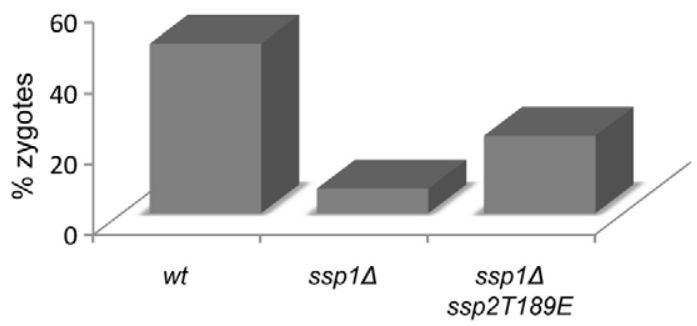

C

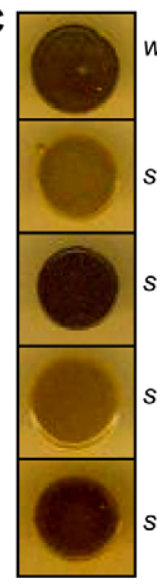

$w t$

$\operatorname{ssp} 2 \Delta$

$\operatorname{ssp} 2+$

ssp2T189A

ssp2T189E

E

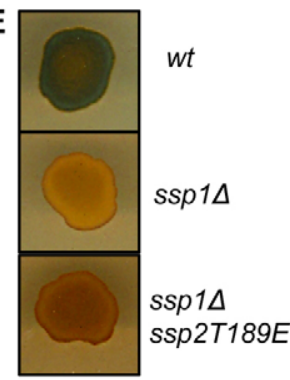

Fig. 7. Activation of AMPK by Ssp2 phosphorylation on Thr189. (A) Wild-type, $s s p 2 \triangle$, $\operatorname{ssp} 2^{+}, \operatorname{ssp} 2(T 189 A)$ and $s s p 2(T 189 E)$ cells were grown to mid-exponential phase in EMM, washed several times and shifted to minimal medium lacking nitrogen. After 4 hours of nitrogen starvation, cell size was analyzed by microscopy. (B,C) Wild-type, $s s p 2 \Delta, s s p 2^{+}$, ssp $2 T 189 A$ and ssp2(T189E) cells of opposite mating types were grown to mid-exponential phase and spotted onto malt extract plates and incubated for 24 hours at $25^{\circ} \mathrm{C}$. The percentage of zygotes was determined by microscopy (B) and spores were stained with iodine vapour (C). (D,E) Mating efficiency in malt extract plates of opposite mating type wild-type, ssp 14 and ssp14 ssp2(T189E) cells. The percentage of zygotes was determined by microscopy (D) and spores were stained with iodine vapour (E). Scale bar: $10 \mu \mathrm{m}$.
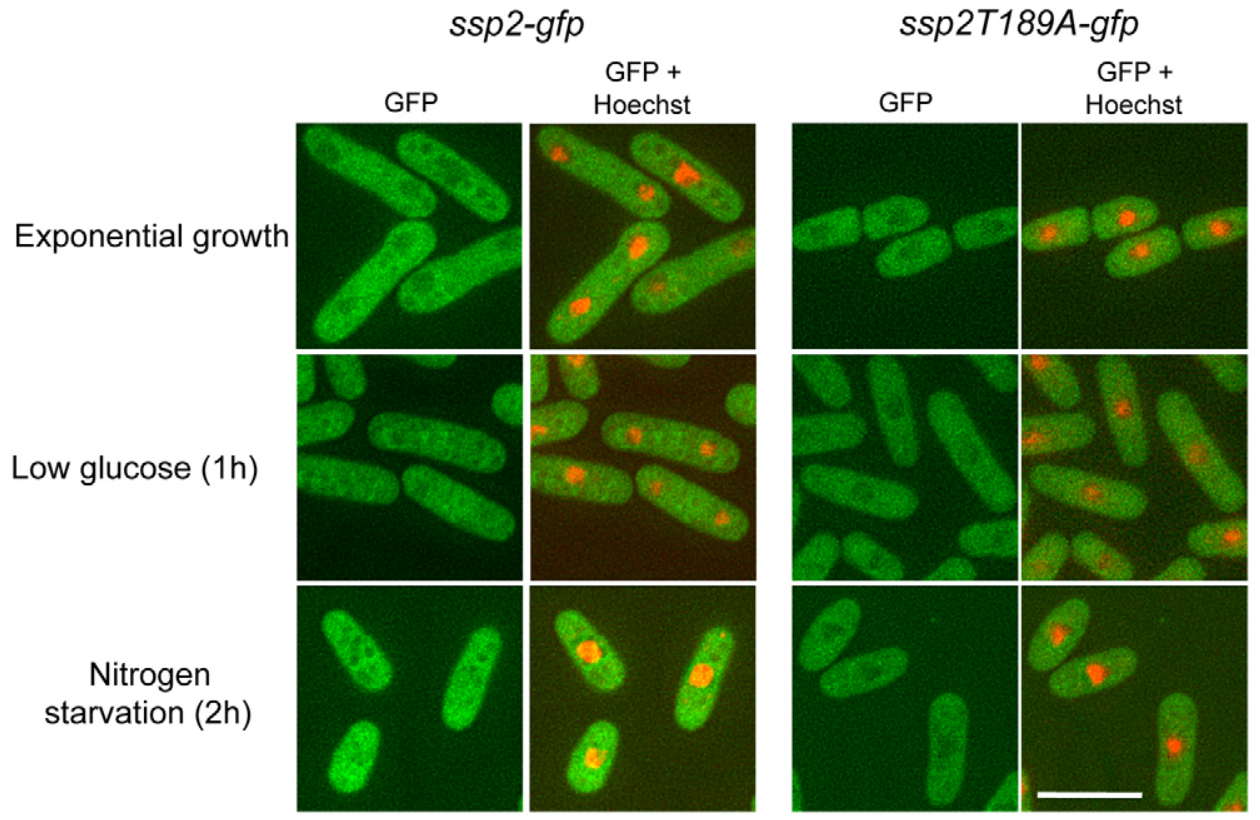

Fig. 8. Nuclear localization of Ssp2 depends on Thr189 phosphorylation. Ssp2-GFP and Ssp2(T189A)-GFP were observed by fluorescence microscopy during exponential growth $(0 \mathrm{~h})$, under low glucose $(1 \mathrm{~h})$ and after nitrogen starvation (2h). Ssp2-GFP localizes to the nucleus upon nitrogen (A) or glucose (B) starvation, whereas Ssp2T189A-GFP localizes only to the cytoplasm. Scale bar: $10 \mu \mathrm{m}$. 
proliferation to avoid nutritional or energy stress that may trigger cell death. When exposed to nutritional stress, fission yeast cells undergo cell cycle exit, G1 arrest and sexual differentiation, followed by meiosis and sporulation, to generate four resistant spores that remain dormant until optimal nutritional conditions have been re-established.

Here we show that AMPK is required for the normal response to nutritional starvation in fission yeast. Fission yeast cells lacking AMPK do not respond by shortening the G2 phase of the cell cycle and entering into mitosis with a reduced cell size (Fig. 1), indicating the importance of AMPK in the adaptation of fission yeast cells to nutritional stress. As a consequence of this abnormal adaptation, cells lacking AMPK activity are unable to arrest in G1 before Start and do not undergo proper sexual differentiation. Microarray analysis (supplementary material Tables S1, S2) indicates that AMPK controls the expression of a large number of genes in response to nutritional stress that are important for arresting cell growth by repressing genes that encode ribosomal proteins, histones, initiation and elongation translation factors, and genes that induce sexual differentiation by activating genes involved in mating, spore biogenesis, etc. It might be concluded that in fission yeast, as in other eukaryotic organisms, AMPK acts as a sensor that promotes cell cycle exit and sexual differentiation in response to a drop in energy levels.
Sexual differentiation is crucial in the adaptation to nitrogen starvation because it allows cells to remain dormant until their original nutritional conditions are restored. The Ste11 transcription factor is crucial in this process. In cells lacking AMPK activity (ssp24), the amount of Ste11 protein present in the nucleus is reduced compared with levels in wild-type cells. Moreover, in our hands, microarray analysis revealed a reduction in Ste11 target genes, indicating low Ste11 activity. Because Ste11 is able to induce its own transcription in cells arrested in G1 with low Cdk activity, the most likely explanation for this reduction in Ste11 levels is that in ssp $2 \Delta$ cells this feedback loop does not work properly.

In all eukaryotic cells, AMPK is present both in the nucleus and the cytoplasm. Environmental stress regulates its nuclear localization in mammals (Kodiha et al., 2007) and in Saccharomyces cerevisiae (Hong and Carlson, 2007). Here we describe that in fission yeast, the three subunits of AMPK are present in the cytoplasm and are excluded from the nucleus during exponential growth in rich medium. Under these conditions, the ATP:AMP ratio is high and AMPK is inactive. After nutritional stress (induced by glucose or nitrogen deprivation), the ATP:AMP ratio falls and AMPK begins to accumulate in the nucleus. This redistribution of AMPK occurs faster in conditions of glucose starvation than of nitrogen starvation (Figs 4, 6), suggesting that the ATP:AMP ratio falls

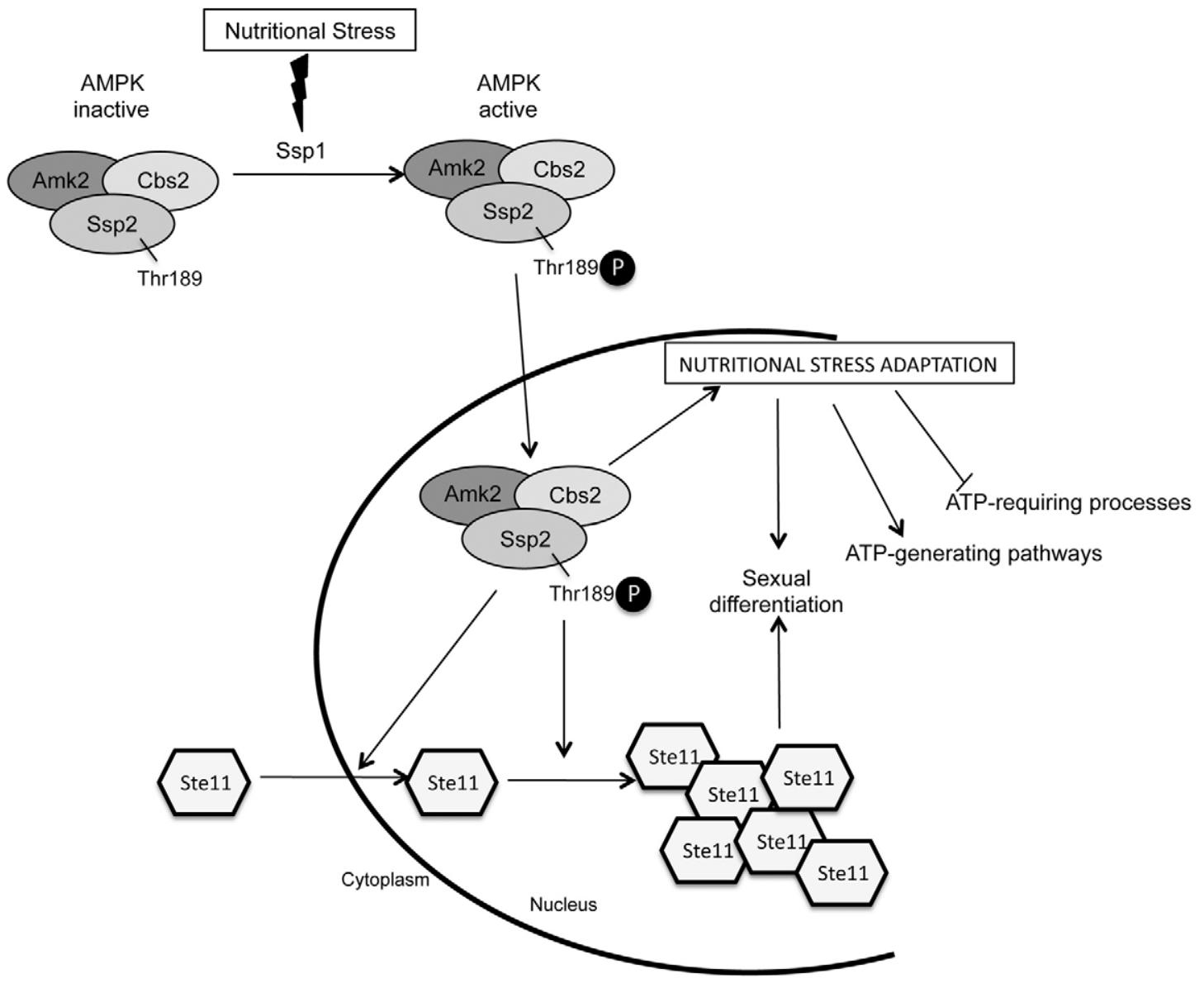

Fig. 9. Hypothetical role of AMPK in fission yeast. During exponential growth, the AMPK complex is inactive. Under nutritional stress, Ssp1 phosphorylates the Ssp2 catalytic subunit on Thr189, activating the complex and triggering its nuclear translocation. Through an unknown molecular mechanism, AMPK allows Ste11 nuclear accumulation to promote sexual differentiation. In addition, AMPK regulates other processes of the adaptation to nutritional stress, inhibiting processes requiring ATP (protein synthesis, mRNA biosynthesis, etc.). 
more dramatically upon glucose withdrawal. To explore the mechanism by which Ssp2 is preferentially translocated to the nucleus under glucose or nitrogen starvation, we searched for nuclear localization signals (NLSs) and nuclear export signals (NESs) in Ssp2. The minimum requirement for an NLS is Lys(Lys/Arg)-X-(Lys/Arg) (Hodel et al., 2001) and two amino acid sequences in Ssp2 met this criterion: Lys237-Lys240 and Lys282-Arg285. Moreover, a putative NES could also be present in the C-terminus of Ssp2. Recently, a functional NES in the C-terminal 22 amino acids of human AMPK a catalytic subunit has been described (Kazgan et al., 2010). The fission yeast Ssp2 C-terminal sequence matches the consensus sequence of highly enriched bulky hydrophobic amino acids ( $\mathrm{f}=\mathrm{Leu}$, Ile, Phe, Val and Met), with the following spacings (f-x-2/3-f-x-2/3-f$\mathrm{x}-\mathrm{f}$ ) in a helix (Kutay and Güttinger, 2005). Further experiments are required to establish the functional significance of these putative NLS and NES sequences in the regulation of $S$. pombe Ssp2 function in response to starvation.

The AMPK $\alpha$-subunit is phosphorylated and activated by phosphorylation on Thr172 by LKB1. Here we show that this residue is conserved in $S$. pombe (Thr189) and that the Ssp1 protein kinase is responsible for this phosphorylation (Fig. 5). When fission yeast cells are under nutritional stress, Ssp1 phosphorylates Ssp2 on Thr189 and promotes its activation. This phosphorylation seems to be required to localize the AMPK complex in the nucleus (Figs 6, 8), because cells lacking Ssp1 or expressing a non-phosphorylatable version of Ssp2(T189A) are unable to accumulate AMPK in the nucleus and, as a consequence, they do not adapt to nutritional stress. It is tempting to speculate that the phosphorylation of AMPK $\alpha$-subunit on Thr189 might regulate the function of the NLS or NES. However, the molecular details and the functional significance of this redistribution of AMPK are currently unknown.

In summary, the data presented in this paper demonstrate the role of AMPK in the adaptation of fission yeast cells to nutritional stress (Fig. 9). When fission yeast cells are exposed to a drop in energy levels, Ssp1 phosphorylates and activates Ssp2, allowing the redistribution of AMPK from the cytoplasm to the nucleus. This response is important to advance cells into mitosis and establish a correct pre-Start G1 cell cycle arrest.

\section{Materials and Methods}

\section{Fission yeast strains and media}

All $S$. pombe strains used in this study are listed in supplementary material Table S3. Standard methods were used for growth, transformation and genetic manipulations (Moreno et al., 1991). The fission yeast tagged strains ssp2-HA, ssp2T189A-HA, ssp2-gfp, amk2-TAP, amk2-gfp, cbs2-TAP, cbs2-gfp, rst2-gfp, rst2-HA and ste11-gfp were generated by a PCR-based method, followed by yeast transformation (Bähler et al., 1998). Except where specifically indicated, all experiments in liquid culture were carried out in Edinburgh Minimal Medium (EMM) containing the required supplements (except when mentioned), starting with a cell density of $2-4 \times 10^{6}$ cells $\mathrm{ml}$, corresponding to the mid-exponential phase of growth. In the case of glucose starvation medium, the concentration of glucose was $0.2 \%$.

\section{Flow cytometry}

Approximately $10^{7}$ cells were collected by centrifugation, fixed in $70 \%$ cold ethanol and processed as described (Moreno et al., 1991). Flow cytometry analysis (FACS) was performed on a Becton-Dickinson FACScan device, using cells stained with propidium iodide. Cell size measurements were accomplished using the forward light scatter (FSC) data of the FACS.

\section{RNA extraction and Northern blots}

Total RNA was isolated by breaking the cells with glass beads in the presence of phenol (Moreno et al., 1991). 5-10 $\mu \mathrm{g}$ of RNA from each sample was separated on a formaldehyde-agarose gel. Northern blotting was carried out using Gene ScreenPlus (NEN, Dupont), following the manufacturer's instructions. DNA probes were labeled with $\left[\alpha_{-}^{32} \mathrm{P}\right] \mathrm{dCTP}$ using the Rediprime II Random Prime Labelling System kit (Amersham)

\section{Protein extraction, immunopurification and western blots}

Protein extracts were obtained using trichloroacetic acid (TCA) extraction, as described previously (Foiani et al., 1994). For immunopurification, $10^{9}$ exponential growing cells were lysed in HB buffer [25 mM MOPS, $60 \mathrm{mM} \beta$ glycerophosphate, $15 \mathrm{mM} \mathrm{MgCl}_{2}, 1 \mathrm{mM}$ DTT, $1 \%$ Triton X-100, $5 \mathrm{mM}$ EGTA and $15 \mathrm{mM}$ pNPP, supplemented with the complete protease inhibitor cocktail tablets (Roche)]. Extracts were incubated with anti-HA 12CA5 antibody for 2 hours with shaking and were pulled down with Protein-A-Sepharose CL-4B (Amersham) for 1 hour. For western blots, 75-100 $\mu \mathrm{g}$ of total protein extract were resolved by $7.5 \%$ SDS-PAGE, transferred to a nitrocellulose filter (Amersham), and probed with mouse anti-HA 12CA5 (Roche Applied Sciences), mouse antiste11 (a gift from Olaf Nielsen, Department of Biology, University of Copenhagen, Denmark), anti-TAP Peroxidase-antiperoxidase soluble complex PAP (Sigma), and mouse anti-tubulin (a gift from Keith Gull, Sir William Dunn School of Pathology, University of Oxford, UK) primary antibodies and, as secondary antibodies, NA 931, anti-mouse IgG, Horseradish Peroxidase (Amersham). Immunoblots were developed using the enhanced chemiluminescence procedure (ECL kit, Amersham). Phos-tag AAL-107 gels (NARD Institute) were used for shifts in the mobility of phosphorylated Ssp2 at a final concentration of $100 \mu \mathrm{M}$ following the protocol suggested by the manufacturer.

\section{Microarrays and data analyses}

Wild-type and ssp2 $\Delta$ cells were exponentially grown in EMM and transferred to EMM without nitrogen for 4 hours. RNA was purified and cleaned using the RNeasy kit (Qiagen). RNA was analyzed with a 2100 Bioanalyzer (Agilent). Double-strand cDNA synthesis kit (Affymetrix) was used for the synthesis and purification of cDNA, and a Nanodrop spectrophotometer was used for cDNA quantification. cDNA was labeled and hybridized with a $S$. pombe Tiling $1.0 \mathrm{FR}$ Array, following the Affymetrix protocol. All the probes included in the arrays were aligned according to the fission yeast genome of the Pombe Sanger Institute Project (http://www.sanger.ac.uk/Projects/S pombe/). To calibrate the sequencespecific probe effect, all data were background-corrected and quantile-normalized. To analyze different levels of transcription, all the normalized data were located in the correct genomic position and were visualized using Artemis (www.sanger.ac. uk/resources/software/artemis/). Initially, we determined the genes regulated by nitrogen by comparing the expression profile of wild-type cells growing exponentially and after nitrogen starvation. We selected those genes in which expression was upregulated or downregulated at least twofold in nitrogen starvation with respect to exponentially growing wild-type cells. Second, to determine which of those genes were not properly upregulated or downregulated in ssp $2 \Delta$ cells under nitrogen starvation, we analyzed the expression profile of those genes previously selected as upregulated or downregulated under nitrogen starvation. We selected those genes whose expression changed at least twofold in $\operatorname{ssp} 2 \Delta$ cells with respect to wild-type cells, in both cases under nitrogen starvation. This way, we identified genes not properly induced in $\operatorname{ssp} 2 \Delta$ cells (twofold downregulated with respect to wild-type cells) or not properly repressed in ssp2 4 cells (twofold upregulated with respect to wild-type cells). The results were organized in Representative Gene Ontology (GO) categories selectively associated with the groups of genes that were regulated by Ssp2 according to the biological process described in Gene DB (http://old.genedb.org/).

\section{Epifluorescence microscopy of GFP fusion proteins}

Epifluorescence microscopy was carried out using an Olympus IX71 fluorescence microscope improved with Delta Vision equipment by Applied Precision. Hoechst 33342 was used to stain DNA. To identify dividing cells, the septum was stained with calcofluor $(5 \mu \mathrm{g} / \mathrm{ml})$. Image J 1.410 software was used to process the images and to measure the length of the cells.

\section{Acknowledgements}

We thank Olaf Nielsen and Keith Gull for antibodies, Angel Prieto for his help with the Deltavision microscope.

\section{Funding}

N.V. is supported by a postdoctoral grant from the Carlos III Institute, Ministerio de Sanidad. Our group is supported by the Ministerio de Economía y Competitividad [grant numbers BFU2008-01808, BFU2011-28274, Consolider CSD2007-00015]; and the Junta de Castilla y León Grupo de Excelencia GR 265.

Supplementary material available online at http://jcs.biologists.org/lookup/suppl/doi:10.1242/jcs.098533/-/DC1 


\section{References}

Adams, J., Chen, Z. P., Van Denderen, B. J., Morton, C. J., Parker, M. W., Witters, L. A., Stapleton, D. and Kemp, B. E. (2004). Intrasteric control of AMPK via the gamma1 subunit AMP allosteric regulatory site. Protein Sci. 13, 155-165.

Bähler, J., Wu, J. Q., Longtine, M. S., Shah, N. G., McKenzie, A., 3rd, Steever, A. B., Wach, A., Philippsen, P. and Pringle, J. R. (1998). Heterologous modules for efficient and versatile PCR-based gene targeting in Schizosaccharomyces pombe. Yeast 14, 943-951.

Bimbó, A., Jia, Y., Poh, S. L., Karuturi, R. K., den Elzen, N., Peng, X., Zheng, L., O'Connell, M., Liu, E. T., Balasubramanian, M. K. et al. (2005). Systematic deletion analysis of fission yeast protein kinases. Eukaryot. Cell 4, 799-813.

Egel, R. and Egel-Mitani, M. (1974). Premeiotic DNA synthesis in fission yeast. Exp. Cell Res. 88, 127-134.

Fantes, P. and Nurse, P. (1977). Control of cell size at division in fission yeast by a growth-modulated size control over nuclear division. Exp. Cell Res. 107, 377-386.

Foiani, M., Marini, F., Gamba, D., Lucchini, G. and Plevani, P. (1994). The B subunit of the DNA polymerase alpha-primase complex in Saccharomyces cerevisiae executes an essential function at the initial stage of DNA replication. Mol. Cell. Biol. 14, 923-933.

Hanyu, Y., Imai, K. K., Kawasaki, Y., Nakamura, T., Nakaseko, Y., Nagao, K., Kokubu, A., Ebe, M., Fujisawa, A., Hayashi, T. et al. (2009). Schizosaccharomyces pombe cell division cycle under limited glucose requires Ssp1 kinase, the putative CaMKK, and Sds23, a PP2A-related phosphatase inhibitor. Genes Cells 14, 539-554.

Hardie, D. G. (2003). Minireview: the AMP-activated protein kinase cascade: the key sensor of cellular energy status. Endocrinology 144, 5179-5183.

Hardie, D. G. (2008). AMPK and Raptor: matching cell growth to energy supply. Mol. Cell 30, 263-265.

Hardie, D. G. (2011). AMP-activated protein kinase: a cellular energy sensor with a key role in metabolic disorders and in cancer. Biochem. Soc. Trans. 39, 1-13.

Hardie, D. G., Hawley, S. A. and Scott, J. W. (2006). AMP-activated protein kinasedevelopment of the energy sensor concept. J. Physiol. 574, 7-15.

Hawley, S. A., Davison, M., Woods, A., Davies, S. P., Beri, R. K., Carling, D. and Hardie, D. G. (1996). Characterization of the AMP-activated protein kinase kinase from rat liver and identification of threonine 172 as the major site at which it phosphorylates AMP-activated protein kinase. J. Biol. Chem. 271, 27879-27887.

Hawley, S. A., Boudeau, J., Reid, J. L., Mustard, K. J., Udd, L., Mäkelä, T. P., Alessi, D. R. and Hardie, D. G. (2003). Complexes between the LKB1 tumor suppressor, STRAD alpha/beta and MO25 alpha/beta are upstream kinases in the AMP-activated protein kinase cascade. J. Biol. 2, 28

Hawley, S. A., Pan, D. A., Mustard, K. J., Ross, L., Bain, J., Edelman, A. M., Frenguelli, B. G. and Hardie, D. G. (2005). Calmodulin-dependent protein kinase kinase-beta is an alternative upstream kinase for AMP-activated protein kinase. Cell Metab. 2, 9-19.

Higuchi, T., Watanabe, Y. and Yamamoto, M. (2002). Protein kinase A regulates sexual development and gluconeogenesis through phosphorylation of the $\mathrm{Zn}$ finger transcriptional activator Rst2p in fission yeast. Mol. Cell. Biol. 22, 1-11.

Hodel, M. R., Corbett, A. H. and Hodel, A. E. (2001). Dissection of a nuclear localization signal. J. Biol. Chem. 276, 1317-1325.

Hong, S. P. and Carlson, M. (2007). Regulation of snf1 protein kinase in response to environmental stress. J. Biol. Chem. 282, 16838-16845.
Hurley, R. L., Anderson, K. A., Franzone, J. M., Kemp, B. E., Means, A. R. and Witters, L. A. (2005). The Ca2+/calmodulin-dependent protein kinase kinases are AMP-activated protein kinase kinases. J. Biol. Chem. 280, 29060-29066.

Jaleel, M., Villa, F., Deak, M., Toth, R., Prescott, A. R., Van Aalten, D. M. and Alessi, D. R. (2006). The ubiquitin-associated domain of AMPK-related kinases regulates conformation and LKB1-mediated phosphorylation and activation. Biochem. J. 394, 545-555.

Jin, X., Townley, R. and Shapiro, L. (2007). Structural insight into AMPK regulation: ADP comes into play. Structure 15, 1285-1295.

Kazgan, N., Williams, T., Forsberg, L. J. and Brenman, J. E. (2010). Identification of a nuclear export signal in the catalytic subunit of AMP-activated protein kinase. Mol. Biol. Cell 21, 3433-3442.

Kjaerulff, S., Andersen, N. R., Borup, M. T. and Nielsen, O. (2007). Cdk phosphorylation of the Ste11 transcription factor constrains differentiation-specific transcription to G1. Genes Dev. 21, 347-359.

Kodiha, M., Rassi, J. G., Brown, C. M. and Stochaj, U. (2007). Localization of AMP kinase is regulated by stress, cell density, and signaling through the MEK->ERK1/2 pathway. Am. J. Physiol. Cell Physiol. 293, C1427-C1436.

Kunitomo, H., Higuchi, T., Iino, Y. and Yamamoto, M. (2000). A zinc-finger protein, Rst2p, regulates transcription of the fission yeast stell(+) gene, which encodes a pivotal transcription factor for sexual development. Mol. Biol. Cell 11, 3205-3217.

Kutay, U. and Güttinger, S. (2005). Leucine-rich nuclear-export signals: born to be weak. Trends Cell Biol. 15, 121-124.

Matsusaka, T., Hirata, D., Yanagida, M. and Toda, T. (1995). A novel protein kinase gene ssp1+ is required for alteration of growth polarity and actin localization in fission yeast. $E M B O J . \mathbf{1 4}, 3325-3338$.

Moreno, S., Klar, A. and Nurse, P. (1991). Molecular genetic analysis of fission yeast Schizosaccharomyces pombe. Methods Enzymol. 194, 795-823.

Nurse, P. and Bissett, Y. (1981). Gene required in G1 for commitment to cell cycle and in G2 for control of mitosis in fission yeast. Nature 292, 558-560.

Qin, J., Kang, W., Leung, B. and McLeod, M. (2003). Ste11p, a high-mobility-group box DNA-binding protein, undergoes pheromone- and nutrient-regulated nuclearcytoplasmic shuttling. Mol. Cell. Biol. 23, 3253-3264.

Sugimoto, A., Iino, Y., Maeda, T., Watanabe, Y. and Yamamoto, M. (1991). Schizosaccharomyces pombe ste11+ encodes a transcription factor with an HMG motif that is a critical regulator of sexual development. Genes Dev. 5, 1990-1999.

Townley, R. and Shapiro, L. (2007). Crystal structures of the adenylate sensor from fission yeast AMP-activated protein kinase. Science 315, 1726-1729.

Vincent, O., Townley, R., Kuchin, S. and Carlson, M. (2001). Subcellular localization of the Snfl kinase is regulated by specific beta subunits and a novel glucose signaling mechanism. Genes Dev. 15, 1104-1114.

Warden, S. M., Richardson, C., O'Donnell, J., Jr, Stapleton, D., Kemp, B. E. and Witters, L. A. (2001). Post-translational modifications of the beta-1 subunit of AMPactivated protein kinase affect enzyme activity and cellular localization. Biochem. J. 354, 275-283.

Woods, A., Dickerson, K., Heath, R., Hong, S. P., Momcilovic, M., Johnstone, S. R., Carlson, M. and Carling, D. (2005). Ca2+/calmodulin-dependent protein kinase kinase-beta acts upstream of AMP-activated protein kinase in mammalian cells. Cell Metab. 2, 21-33.

Xiao, B., Sanders, M. J., Underwood, E., Heath, R., Mayer, F. V., Carmena, D., Jing, C., Walker, P. A., Eccleston, J. F., Haire, L. F. et al. (2011). Structure of mammalian AMPK and its regulation by ADP. Nature 472, 230-233. 